\title{
Generalized Kato decomposition and essential spectra
}

\author{
Miloš D. Cvetković, Snežana Č. Živković-Zlatanović*
}

\begin{abstract}
Let $\mathbf{R}$ denote any of the following classes: upper (lower) semi-Fredholm operators, Fredholm operators, upper (lower) semi-Weyl operators, Weyl operators, upper (lower) semi-Browder operators, Browder operators. For a bounded linear operator $T$ on a Banach space $X$ we prove that $T=T_{M} \oplus T_{N}$ with $T_{M} \in \mathbf{R}$ and $T_{N}$ quasinilpotent (nilpotent) if and only if $T$ admits a generalized Kato decomposition ( $T$ is of Kato type) and 0 is not an interior point of the corresponding spectrum $\sigma_{\mathbf{R}}(T)=\{\lambda \in \mathbb{C}: T-\lambda \notin \mathbf{R}\}$. In addition, we show that every non-isolated boundary point of the spectrum $\sigma_{\mathbf{R}}(T)$ belongs to the generalized Kato spectrum of $T$.
\end{abstract}

2010 Mathematics subject classification: 47A53, 47A10.

Key words and phrases: generalized Kato decomposition, generalized Kato spectrum, upper and lower semi-Fredholm, semi-Weyl and semi-Browder operators.

\section{Introduction}

Given a Banach space $X$ and an operator $T \in L(X), T$ is Drazin (generalized Drazin) invertible if and only if $T$ is the direct sum of an invertible and a nilpotent (quasinilpotent) operator [18, 22, 19]. M. Berkani proved that $T$ is B-Fredholm if and only if $T=T_{1} \oplus T_{2}$ with $T_{1}$ Fredholm and $T_{2}$ nilpotent [5. Theorem 2.7]. Very recently, pseudo B-Fredholm and pseudo B-Weyl operators were defined in a sense that $T$ is pseudo B-Fredholm (pseudo BWeyl) if $T=T_{1} \oplus T_{2}$, where $T_{1}$ is Fredholm (Weyl) and $T_{2}$ is quasinilpotent [8, 26. In accordance with these observations it is natural to study various types of the direct sums. Namely, let $\mathbf{R}$ denote any of the following

${ }^{*}$ The authors are supported by the Ministry of Education, Science and Technological Development, Republic of Serbia, grant no. 174007. 
classes: upper (lower) semi-Fredholm operators, Fredholm operators, upper (lower) semi-Weyl operators, Weyl operators, upper (lower) semi-Browder operators, Browder operators, bounded below operators, surjective operators, invertible operators. The main objective of this article is to provide necessary and sufficient conditions for an operator $T \in L(X)$ to be the direct sum of an operator $T_{1} \in \mathbf{R}$ and a quasinilpotent (nilpotent) operator $T_{2}$. We approach the problem by using mainly the gap theory and the advantage of our method lies in the fact that it enables us to investigate all cases (for all $\mathbf{R}$ mentioned above) at the same time.

We are also able to derive some recent results in a different way. Namely, P. Aiena and E. Rosas prove in [2] that if $T \in L(X)$ is of Kato type, then $T$ $\left(T^{\prime}\right)$ has the SVEP at 0 if and only if the approximate point (surjective) spectrum of $T$ does not cluster at 0 . Q. Jiang and H. Zhong extend this result to the operators that admit a generalized Kato decomposition [14. They show that if $T$ admits a GKD, $T\left(T^{\prime}\right)$ has the SVEP at 0 if and only if 0 is not an accumulation point of its approximate point (surjective) spectrum, and it is exactly when 0 is not an interior point of the approximate point (surjective) spectrum of $T$. We extend these results to the cases of the essential spectra. Namely, we show for every class $\mathbf{R}$ mentioned earlier that if $T$ admits a GKD ( $T$ is of Kato type), 0 is not an accumulation point of the spectrum $\sigma_{\mathbf{R}}(T)=$ $\{\lambda \in \mathbb{C}: T-\lambda \notin \mathbf{R}\}$ if and only if 0 is not an interior point of $\sigma_{\mathbf{R}}(T)$. In particular, if $\mathbf{R} \in$ \{bounded below operators, surjective operators $\}$ we obtain the result of $\mathrm{Q}$. Jiang and $\mathrm{H}$. Zhong. What is more, for $T \in L(X)$ it is proved that if 0 is a boundary point of $\sigma_{\mathbf{R}}(T), T$ admits a GKD ( $T$ is of Kato type) if and only if $T$ is the direct sum of an operator that belongs to the class $\mathbf{R}$ and a quasinilpotent (nilpotent) operator. In the special case of invertible operators from this we derive the result of P. Aiena and E. Rosas (the result of $\mathrm{Q}$. Jiang and $\mathrm{H}$. Zhong) which says that if 0 is a boundary point of the spectrum of $T \in L(X)$, then $T$ is of Kato type ( $T$ admits a GKD) if and only if $T$ is Drazin (generalized Drazin) invertible; see [2, Theorem 2.9] and [14, Theorem 3.8]. It is worth mentioning that this result of P. Aiena and E. Rosas is an improvement of an earlier result of M. Berkani which shows that if 0 is isolated in the spectrum of $T$, then $T$ is a B-Weyl operator if and only if it is Drazin invertible [7, Theorem 4.2].

In Section 2 we set up terminology and recall necessary facts. Our main results are established in Section 3. Given an operator $T \in L(X), X$ is a Banach space, we prove that $T=T_{M} \oplus T_{N}$ with $T_{M} \in \mathbf{R}$ and $T_{N}$ quasinilpotent if and only if $T$ admits a generalized Kato decomposition and 0 is not an interior point of $\sigma_{\mathbf{R}}(T)$. What is more, the condition that $T$ admits a generalized Kato decomposition cannot be omitted and it will be demonstrated 
by an example. We also show that necessary and sufficient for $T \in L(X)$ to be the direct sum $T=T_{M} \oplus T_{N}$ with $T_{M} \in \mathbf{R}$ and $T_{N}$ quasinilpotent is that $T$ admits a generalized Kato decomposition and that 0 is not an accumulation point of the spectrum $\sigma_{\mathbf{R}}(T)$. If $\mathbf{R}$ is the class of invertible operators the condition that $T$ admits a GKD is unnecessary [19, Theorem 7.1]. If $\sigma_{\mathbf{R}}(T)$ is any of the essential spectra we demonstrate by an example that this condition can not be excluded. If $\mathbf{R}$ represents the class of bounded below or surjective operators, we are not sure whether this condition can be omitted and it is an open question. Also, it is possible to obtain some results related to the B-Fredholm and B-Weyl operators. In particular, an operator $T$ is B-Fredholm (B-Weyl) if and only if it is of Kato type and if 0 is not an interior point of its Fredholm (Weyl spectrum).

In the fourth section some applications are presented. We prove that every boundary point of $\sigma_{\mathbf{R}}(T)$, where $\mathbf{R}$ is any of the classes mentioned above, which is also an accumulation point of $\sigma_{\mathbf{R}}(T)$ belongs to the generalized Kato spectrum. In particular, if $\mathbf{R}$ is the class of invertible operators we obtain [14, Corollary 3.6]. If $T \in L(X)$, let $\sigma_{\mathbf{g D R}}(T)$ be the set of all $\lambda \in \mathbb{C}$ such that $T-\lambda$ can not be represented as the direct sum of an operator from the class $\mathbf{R}$ and a quasinilpotent operator. We show that the connected hull of the spectrum $\sigma_{\mathbf{g D R}}(T)$ coincide with the connected hull of the generalized Kato spectrum for every class R. In particular, the connected hulls of the generalized Drazin spectrum and the generalized Kato spectrum are equal. Moreover, the connected hulls of the B-Fredholm, B-Weyl, Drazin and of the Kato type spectrum are equal.

\section{Preliminaries}

Let $\mathbb{N}\left(\mathbb{N}_{0}\right)$ denote the set of all positive (non-negative) integers, and let $\mathbb{C}$ denote the set of all complex numbers. Let $X$ be an infinite dimensional Banach space and let $L(X)$ be the Banach algebra of all bounded linear operators acting on $X$. Given $T \in L(X)$, we denote by $N(T), R(T)$ and $\sigma(T)$, the kernel, the range and the spectrum of $T$, respectively. In addition, $\alpha(T)$ and $\beta(T)$ will stand for nullity and defect of $T$. The space of bounded linear functionals on $X$ is denoted by $X^{\prime}$. If $K \subset \mathbb{C}$, then $\partial K$ is the boundary of $K$, acc $K$ is the set of accumulation points of $K$, iso $K=K \backslash$ acc $K$ and int $K$ is the set of interior points of $K$. The group of all invertible operators is denoted by $L(X)^{-1}$.

Recall that $T$ is said to be nilpotent when $T^{n}=0$ for some $n \in \mathbb{N}$, while $T$ is quasinilpotent if $\left\|T^{n}\right\|^{1 / n} \rightarrow 0$, that is $T-\lambda \in L(X)^{-1}$ for all complex 
$\lambda \neq 0$. An operator $T \in L(X)$ is bounded below if there exists some $c>0$ such that $c\|x\| \leq\|T x\|$ for every $x \in X$. Let $\mathcal{M}(X)$ denote the set of all bounded below operators, and let $\mathcal{Q}(X)$ denote the set of all surjective operators. The approximate point spectrum of $T \in L(X)$ is defined by

$$
\sigma_{a p}(T)=\{\lambda \in \mathbb{C}: T-\lambda \text { is not bounded below }\}
$$

and the surjective spectrum is defined by

$$
\sigma_{s u}(T)=\{\lambda \in \mathbb{C}: T-\lambda \text { is not surjective }\} .
$$

An operator $T \in L(X)$ is Kato if $R(T)$ is closed and $N(T) \subset R\left(T^{n}\right), n \in \mathbb{N}_{0}$. If $R(T)$ is closed and $\alpha(T)<\infty$, then $T \in L(X)$ is said to be upper semiFredholm. An operator $T \in L(X)$ is lower semi-Fredholm if $\beta(T)<\infty$. The set of upper semi-Fredholm operators (lower semi-Fredholm operators) is denoted by $\Phi_{+}(X)\left(\Phi_{-}(X)\right)$. If $T$ is upper or lower semi-Fredholm operator then the index of $T$ is defined as $\operatorname{ind}(T)=\alpha(T)-\beta(T)$. An operator $T$ is Fredholm if both $\alpha(T)$ and $\beta(T)$ are finite. We will denote by $\Phi(X)$ the set of Fredholm operators. The sets of upper semi-Weyl, lower semi Weyl and $W e y l$ operators are defined by $\mathcal{W}_{+}(X)=\left\{T \in \Phi_{+}(X): \operatorname{ind}(T) \leq 0\right\}$, $\mathcal{W}_{-}(X)=\left\{T \in \Phi_{-}(X): \operatorname{ind}(T) \geq 0\right\}$ and $\mathcal{W}(X)=\{T \in \Phi(X): \operatorname{ind}(T)=$ $0\}$, respectively. B-Fredholm and B-Weyl operators were introduced and studied by M. Berkani [5, 6, 7]. An operator $T \in L(X)$ is said to be BFredholm (B-Weyl) if there is $n \in \mathbb{N}$ such that $R\left(T^{n}\right)$ is closed and the restriction $T_{n} \in L\left(R\left(T^{n}\right)\right)$ of $T$ to $R\left(T^{n}\right)$ is Fredholm (Weyl). The BFredholm and the B-Weyl spectrum of $T$ are defined by

$$
\begin{aligned}
\sigma_{B \Phi}(T) & =\{\lambda \in \mathbb{C}: T-\lambda \text { is not B-Fredholm }\} \\
\sigma_{B \mathcal{W}}(T) & =\{\lambda \in \mathbb{C}: T-\lambda \text { is not B-Weyl }\}, \text { respectively. }
\end{aligned}
$$

Recall that $T \in L(X)$ is said to be Riesz operator, if $T-\lambda \in \Phi(X)$ for every non-zero $\lambda \in \mathbb{C}$.

The ascent of $T$ is defined as $\operatorname{asc}(T)=\inf \left\{n \in \mathbb{N}_{0}: N\left(T^{n}\right)=N\left(T^{n+1}\right)\right\}$, and descent of $T$ is defined as $\operatorname{dsc}(T)=\inf \left\{n \in \mathbb{N}_{0}: R\left(T^{n}\right)=R\left(T^{n+1}\right)\right\}$, where the infimum over the empty set is taken to be infinity. An operator $T \in L(X)$ is upper semi-Browder if $T$ is upper semi-Fredholm and $\operatorname{asc}(T)<$ $\infty$. If $T \in L(X)$ is lower semi-Fredholm and $\operatorname{dsc}(T)<\infty$, then $T$ is lower semi-Browder. Let $\mathcal{B}_{+}(X)\left(\mathcal{B}_{-}(X)\right)$ denote the set of all upper (lower) semiBrowder operators. The set of Browder operators is defined by $\mathcal{B}(X)=$ $\mathcal{B}_{+}(X) \cap \mathcal{B}_{-}(X)$.

If $M$ is a subspace of $X$ such that $T(M) \subset M, T \in L(X)$, it is said that $M$ is $T$-invariant. We define $T_{M}: M \rightarrow M$ as $T_{M} x=T x, x \in M$. If $M$ 
and $N$ are two closed $T$-invariant subspaces of $X$ such that $X=M \oplus N$, we say that $T$ is completely reduced by the pair $(M, N)$ and it is denoted by $(M, N) \in \operatorname{Red}(T)$. In this case we write $T=T_{M} \oplus T_{N}$ and say that $T$ is the direct sum of $T_{M}$ and $T_{N}$.

An operator $T \in L(X)$ is said to admit a generalized Kato decomposition, abbreviated as GKD, if there exists a pair $(M, N) \in \operatorname{Red}(T)$ such that $T_{M}$ is Kato and $T_{N}$ is quasinilpotent. A relevant case is obtained if we assume that $T_{N}$ is nilpotent. In this case $T$ is said to be of Kato type. An operator is said to be essentially Kato if it admits a $\operatorname{GKD}(M, N)$ such that $N$ is finite-dimensional. If $T$ is essentially Kato then $T_{N}$ is nilpotent, since every quasinilpotent operator on a finite dimensional space is nilpotent. The classes $\Phi_{+}(X), \Phi_{-}(X), \Phi(X), \mathcal{B}_{+}(X), \mathcal{B}_{-}(X), \mathcal{B}(X), \mathcal{W}_{+}(X), \mathcal{W}_{-}(X)$ and $\mathcal{W}(X)$ belong to the class of essentially Kato operators [24, Theorem 16.21]. For $T \in L(X)$, the Kato spectrum, the essentially Kato spectrum, the Kato type spectrum and the generalized Kato spectrum are defined by

$$
\begin{aligned}
\sigma_{K}(T) & =\{\lambda \in \mathbb{C}: T-\lambda \text { is not Kato }\}, \\
\sigma_{e K}(T) & =\{\lambda \in \mathbb{C}: T-\lambda \text { is not essentially Kato }\}, \\
\sigma_{K t}(T) & =\{\lambda \in \mathbb{C}: T-\lambda \text { is not of Kato type }\}, \\
\sigma_{g K}(T) & =\{\lambda \in \mathbb{C}: T-\lambda \text { does not admit a GKD }\},
\end{aligned}
$$

respectively. Clearly,

$$
\sigma_{g K}(T) \subset \sigma_{K t}(T) \subset \sigma_{e K}(T) \subset \sigma_{K}(T) \subset \sigma_{a p}(T) \cap \sigma_{s u}(T) .
$$

The quasinilpotent part $H_{0}(T)$ of an operator $T \in L(X)$ is defined by

$$
H_{0}(T)=\left\{x \in X: \lim _{n \rightarrow+\infty}\left\|T^{n} x\right\|^{1 / n}=0\right\} .
$$

It is easy to verify that $H_{0}(T)=\{0\}$ if $T$ is bounded below. An operator $T \in L(X)$ is quasinilpotent if and only if $H_{0}(T)=X$ [1, Theorem 1.68].

The analytical core of $T$, denoted by $K(T)$, is the set of all $x \in X$ for which there exist $c>0$ and a sequence $\left(x_{n}\right)_{n}$ in $X$ satisfying

$$
T x_{1}=x, T x_{n+1}=x_{n} \text { for all } n \in \mathbb{N},\left\|x_{n}\right\| \leq c^{n}\|x\| \text { for all } n \in \mathbb{N} .
$$

If $T$ is surjective, then $K(T)=X$ [1, Theorem 1.22].

An operator $T \in L(X)$ is said to be generalized Drazin invertible, if there exists $B \in L(X)$ such that

$$
T B=B T, \quad B T B=B, \quad T B T-T \text { is quasinilpotent } .
$$


The generalized Drazin spectrum of $T \in L(X)$ is defined by

$$
\sigma_{g D}(T)=\{\lambda \in \mathbb{C}: T-\lambda \text { is not generalized Drazin invertible }\} .
$$

The equivalent conditions to the existence of generalized Drazin inverse of a bounded operator are collected in the following theorem.

Theorem 2.1 (see [19], 23], 25], 20], 11]). Let $T \in L(X)$. The following conditions are equivalent:

(i) $T$ is generalized Drazin invertible;

(ii) There exists a bounded projection $P$ on $X$ which commutes with $T$ such that $T+P$ is invertible and $T P$ is quasinilpotent;

(iii) $0 \notin \operatorname{acc} \sigma(T)$;

(iv) There is a bounded projection $P$ on $X$ such that $R(P)=H_{0}(T)$ and $N(T)=K(T)$;

(v) There exists $(M, N) \in \operatorname{Red}(T)$ such that $T_{M}$ is invertible and $T_{N}$ is quasinilpotent;

(vi) $X=K(T) \oplus H_{0}(T)$ with at least one of the component spaces closed.

For a subspace $M$ of $X$ its annihilator $M^{\perp}$ is defined by

$$
M^{\perp}=\left\{f \in X^{\prime}: f(x)=0 \text { for all } x \in M\right\} .
$$

Recall that if $M$ is closed, then

$$
\operatorname{dim} M^{\perp}=\operatorname{codim} M .
$$

Let $M$ and $L$ be two subspaces of $X$ and let

$$
\delta(M, L)=\sup \{\operatorname{dist}(u, L): u \in M,\|u\|=1\},
$$

in the case that $M \neq\{0\}$, otherwise we define $\delta(\{0\}, L)=0$ for any subspace $L$. The gap between $M$ and $L$ is defined by

$$
\hat{\delta}(M, L)=\max \{\delta(M, L), \delta(L, M)\} .
$$

It is known that [24, corollary 10.10]

$$
\hat{\delta}(M, L)<1 \Longrightarrow \operatorname{dim} M=\operatorname{dim} L .
$$

If $M$ and $L$ are closed subspaces of $X$, then [24, Theorem 10.8]

$$
\hat{\delta}\left(M^{\perp}, L^{\perp}\right)=\hat{\delta}(M, L) .
$$


Therefore, for closed subspaces $M$ and $L$ of $X$, according to (2.2), (2.3) and (2.4), there is implication

$$
\hat{\delta}(M, L)<1 \Longrightarrow \operatorname{codim} M=\operatorname{codim} L .
$$

We use the following notation.

\begin{tabular}{|c|c|c|}
\hline $\mathbf{R}_{\mathbf{1}}=\Phi_{+}(X)$ & $\mathbf{R}_{\mathbf{2}}=\Phi_{-}(X)$ & $\mathbf{R}_{\mathbf{3}}=\Phi(X)$ \\
\hline $\mathbf{R}_{\mathbf{4}}=\mathcal{W}_{+}(X)$ & $\mathbf{R}_{\mathbf{5}}=\mathcal{W}_{-}(X)$ & $\mathbf{R}_{\mathbf{6}}=\mathcal{W}(X)$ \\
\hline $\mathbf{R}_{\mathbf{7}}=\mathcal{B}_{+}(X)$ & $\mathbf{R}_{\mathbf{8}}=\mathcal{B}_{-}(X)$ & $\mathbf{R}_{\mathbf{9}}=\mathcal{B}(X)$ \\
\hline $\mathbf{R}_{\mathbf{1 0}}=\mathcal{M}(X)$ & $\mathbf{R}_{\mathbf{1 1}}=\mathcal{Q}(X)$ & $\mathbf{R}_{\mathbf{1 2}}=L(X)^{-1}$ \\
\hline
\end{tabular}

The sets $\mathbf{R}_{i}, 1 \leq i \leq 12$, are open in $L(X)$ and contain $L(X)^{-1}$ (for the openness of the set of upper (lower) semi-Browder operators see [21, Satz 4]). The spectra $\sigma_{\mathbf{R}_{i}}(T)=\left\{\lambda \in \mathbb{C}: T-\lambda \notin \mathbf{R}_{i}\right\}, 1 \leq i \leq 12$, are non-empty and compact subsets of $\mathbb{C}$. We write $\sigma_{\mathbf{R}_{1}}(T)=\sigma_{\Phi_{+}}(T), \sigma_{\mathbf{R}_{2}}(T)=\sigma_{\Phi_{-}}(T)$, etc, and $\rho_{\Phi_{+}}(T)=\mathbb{C} \backslash \sigma_{\Phi_{+}}(T), \rho_{\Phi_{-}}(T)=\mathbb{C} \backslash \sigma_{\Phi_{-}}(T)$, etc. In particular, $\sigma_{\mathbf{R}_{10}}(T)=\sigma_{a p}(T), \sigma_{\mathbf{R}_{11}}(T)=\sigma_{s u}(T), \rho_{a p}(T)=\mathbb{C} \backslash \sigma_{a p}(T)$ and $\rho_{s u}(T)=$ $\mathbb{C} \backslash \sigma_{s u}(T)$. We consider the following classes of bounded linear operators:

$\mathbf{g D R}_{i}=\left\{T \in L(X): \begin{array}{c}\text { there exists }(M, N) \in \operatorname{Red}(T) \text { such that } \\ T_{M} \in \mathbf{R}_{i} \text { and } T_{N} \text { is quasinilpotent }\end{array}\right\}, \quad 1 \leq i \leq 12$.

If $T_{N}$ mentioned in this definition is nilpotent then it is said that $T$ belongs to the class $\mathbf{D R}_{i}, 1 \leq i \leq 12$. It is clear that $\mathbf{R}_{i} \subset \mathbf{D} \mathbf{R}_{i} \subset \mathbf{g D R}, 1 \leq i \leq 12$.

We shall say that $T \in L(X)$ is generalized Drazin upper semi-Fredholm (resp. generalized Drazin lower semi-Fredholm, generalized Drazin Fredholm, generalized Drazin upper semi-Weyl, generalized Drazin lower semiWeyl, generalized Drazin Weyl, generalized Drazin bounded below, generalized Drazin surjective) if $T \in \mathbf{g D} \Phi_{+}(X)$ (resp. $\mathbf{g D} \Phi_{-}(X), \mathbf{g D} \mathbf{\Phi}(X)$, $\left.\operatorname{gD} \mathcal{W}_{+}(X), \operatorname{gD} \mathcal{W}_{-}(X), \operatorname{gD} \mathcal{W}(X), \operatorname{gD} \mathcal{M}(X), \operatorname{gD} \mathcal{Q}(X)\right)$. The reason for introducing these names is that all these classes generalize the class of generalized Drazin invertible operators and, as we will see, may be characterized in a similar way as the class of generalized Drazin invertible operators. We remark that pseudo B-Fredholm operators and generalized Drazin Fredholm operators coincide, as well as, pseudo B-Weyl operators and generalized Drazin Weyl operators.

The following technical lemma will be useful in the sequel.

Lemma 2.2. Let $T \in L(X)$ and $(M, N) \in \operatorname{Red}(T)$. The following statements hold: 
(i) $T \in \mathbf{R}_{i}$ if and only if $T_{M} \in \mathbf{R}_{i}$ and $T_{N} \in \mathbf{R}_{i}, 1 \leq i \leq 3$ or $7 \leq i \leq 12$, and in that case $\operatorname{ind}(T)=\operatorname{ind}\left(T_{M}\right)+\operatorname{ind}\left(T_{N}\right)$;

(ii) If $T_{M} \in \mathbf{R}_{i}$ and $T_{N} \in \mathbf{R}_{i}$, then $T \in \mathbf{R}_{i}, 4 \leq i \leq 6$.

(iii) If $T \in \mathbf{R}_{i}$ and $T_{N}$ is Weyl, then $T_{M} \in \mathbf{R}_{i}, 4 \leq i \leq 6$.

Proof. (i): From the equalities $N(T)=N\left(T_{M}\right) \oplus N\left(T_{N}\right)$ and $R(T)=$ $R\left(T_{M}\right) \oplus R\left(T_{N}\right)$ it follows that $\alpha(T)=\alpha\left(T_{M}\right)+\alpha\left(T_{N}\right)$ and $\beta(T)=\beta\left(T_{M}\right)+$ $\beta\left(T_{N}\right)$. It implies that $\alpha(T)<\infty$ if and only if $\alpha\left(T_{M}\right)<\infty$ and $\alpha\left(T_{N}\right)<\infty$, and also, $\beta(T)<\infty$ if and only if $\beta\left(T_{M}\right)<\infty$ and $\beta\left(T_{N}\right)<\infty$. It is known that $R(T)$ is closed if and only if $R\left(T_{M}\right)$ and $R\left(T_{N}\right)$ are closed [14, Lemma 3.3]. Therefore $T$ is bounded below (surjective, upper semi-Fredholm, lower semi-Fredholm) if and only if $T_{M}$ and $T_{N}$ are bounded below (surjective, upper semi-Fredholm, lower semi-Fredholm), and in that case $\operatorname{ind}(T)=$ $\alpha(T)-\beta(T)=\left(\alpha\left(T_{M}\right)+\alpha\left(T_{N}\right)\right)-\left(\beta\left(T_{M}\right)+\beta\left(T_{N}\right)\right)=\operatorname{ind}\left(T_{M}\right)+\operatorname{ind}\left(T_{M}\right)$.

Since $N\left(T^{n}\right)=N\left(T_{M}^{n}\right) \oplus N\left(T_{N}^{n}\right)$, for every $n \in \mathbb{N}$, we conclude that $\operatorname{asc}(T)<\infty$ if and only if $\operatorname{asc}\left(T_{M}\right)<\infty$ and $\operatorname{asc}\left(T_{N}\right)<\infty$, with $\operatorname{asc}(T)=$ $\max \left\{\operatorname{asc}\left(T_{M}\right), \operatorname{asc}\left(T_{N}\right)\right\}$. Similarly, as $R\left(T^{n}\right)=R\left(T_{M}^{n}\right) \oplus R\left(T_{N}^{n}\right), n \in \mathbb{N}$, we get that $\operatorname{dsc}(T)<\infty$ if and only if $\operatorname{dsc}\left(T_{M}\right)<\infty$ and $\operatorname{dsc}\left(T_{N}\right)<\infty$, with $\operatorname{dsc}(T)=\max \left\{\operatorname{dsc}\left(T_{M}\right), \operatorname{dsc}\left(T_{N}\right)\right\}$.

(ii): Follows from (i).

(iii): Suppose that $T \in \mathcal{W}_{+}(X)$ and that $T_{N}$ is Weyl. According to (i) it follows that $T_{M} \in \Phi_{+}(X)$ and $\operatorname{ind}\left(T_{M}\right)=\operatorname{ind}\left(T_{M}\right)+\operatorname{ind}\left(T_{N}\right)=\operatorname{ind}(T) \leq 0$. Thus $T_{M}$ is upper semi-Weyl. The cases $i=5$ and $i=6$ can be proved similarly.

\section{Main results}

We start with the result which is essential for our work.

Proposition 3.1. Let $T \in L(X)$. Then the following implications hold:

(i) If $T$ is Kato and $0 \in \operatorname{acc} \rho_{\Phi_{+}}(T)\left(0 \in \operatorname{acc} \rho_{\Phi_{-}}(T)\right)$, then $T$ is upper (lower) semi-Fredholm;

(ii) If $T$ is Kato and $0 \in \operatorname{acc} \rho_{\mathcal{W}_{+}}(T)\left(0 \in \operatorname{acc} \rho_{\mathcal{W}_{-}}(T)\right)$, then $T$ is upper (lower) semi-Weyl;

(iii) If $T$ is Kato and $0 \in \operatorname{acc} \rho_{a p}(T)\left(0 \in \operatorname{acc} \rho_{s u}(T)\right)$, then $T$ is bounded below (surjective);

(iv) If $T$ is Kato and $0 \in \operatorname{acc} \rho_{\mathcal{B}_{+}}(T)\left(0 \in \operatorname{acc} \rho_{\mathcal{B}_{-}}(T)\right)$, then $T$ is bounded below (surjective). 
Proof. (i): Suppose that $T$ is Kato. According to [24, Corollary 12.4], $T-\lambda$ is Kato for all $\lambda$ in a neighborhood of 0 . From [24, Theorem 12.2] it follows that $\lim _{\lambda \rightarrow 0} \hat{\delta}(N(T), N(T-\lambda))=0$ and $\lim _{\lambda \rightarrow 0} \hat{\delta}(R(T-\lambda), R(T))=0$ and hence, there exists $\delta>0$ such that for all $|\lambda|<\delta$ it holds that

$$
\begin{gathered}
\hat{\delta}(N(T), N(T-\lambda))<1, \quad \hat{\delta}(R(T-\lambda), R(T))<1, \\
T-\lambda \text { is Kato, }
\end{gathered}
$$

and hence $R(T-\lambda)$ is closed. By [24, Corollary 10.10], (3.1), (3.2) and (2.5), for all $|\lambda|<\delta$ the following holds

$$
\operatorname{dim} N(T-\lambda)=\operatorname{dim} N(T) \text { i } \operatorname{codim} R(T)=\operatorname{codim} R(T-\lambda) .
$$

Suppose now that $0 \in \operatorname{acc} \rho_{\Phi_{+}}(T)\left(0 \in \operatorname{acc} \rho_{\Phi_{-}}(T)\right)$. Then there exists $\mu$ such that $0<|\mu|<\delta$ and $T-\mu$ is upper semi-Fredholm (lower semiFredholm), and so from (3.3) it follows that $\operatorname{dim} N(T)=\operatorname{dim} N(T-\mu)<+\infty$ $(\operatorname{codim} R(T)=\operatorname{codim} R(T-\mu)<+\infty)$. Consequently, $T$ is upper semiFredholm (lower semi-Fredholm).

(ii): Suppose that $T$ is Kato and $0 \in \operatorname{acc} \rho_{\mathcal{W}_{+}}(T)$. Then there exists $\delta>0$ such that (3.2) and (3.3) hold for all $|\lambda|<\delta$. Since $0 \in \operatorname{acc} \rho_{\mathcal{W}_{+}}(T)$, there exists $\mu$ such that $0<|\mu|<\delta$ and $T-\mu$ is upper semi-Weyl. From (3.3) it follows that $\operatorname{dim} N(T)=\operatorname{dim} N(T-\mu)<\infty, \operatorname{codim} R(T)=\operatorname{codim} R(T-\mu)$ and $\operatorname{ind}(T)=\operatorname{dim} N(T)-\operatorname{codim} R(T)=\operatorname{dim} N(T-\mu)-\operatorname{codim} R(T-\mu)=$ $\operatorname{ind}(T-\mu) \leq 0$ and so $T$ is upper semi-Weyl. The proof of $T$ being lower semi-Weyl if $T$ is Kato and $0 \in \operatorname{acc} \rho_{\mathcal{W}_{-}}(T)$ is analogous.

(iii): Let $T$ be Kato and let $0 \in \operatorname{acc} \rho_{a p}(T)\left(0 \in \operatorname{acc} \rho_{s u}(T)\right)$. Dealing as in the previous part of the proof we find $\delta>0$ and $\mu \in \mathbb{C}, 0<|\mu|<\delta$, such that (3.2) and (3.3) hold for all $|\lambda|<\delta$ and $T-\mu$ is bounded below (surjective). From (3.3) it follows that $\operatorname{dim} N(T)=\operatorname{dim} N(T-\mu)=0$ $(\operatorname{codim} R(T)=\operatorname{codim} R(T-\mu)=0)$, and so $T$ is bounded below (surjective). (iv): Let $T$ be Kato and let $0 \in \operatorname{acc} \rho_{\mathcal{B}_{+}}(T)\left(0 \in \operatorname{acc} \rho_{\mathcal{B}_{-}}(T)\right)$. Then there exists $\delta>0$ such that (3.2) and (3.3) hold for all $|\lambda|<\delta$. From $0 \in$ $\operatorname{acc} \rho_{\mathcal{B}_{+}}(T) \quad\left(0 \in \operatorname{acc} \rho_{\mathcal{B}_{-}}(T)\right)$ it follows that there exists $\mu$ such that $0<$ $|\mu|<\delta$ and $T-\mu$ is upper semi-Browder (lower semi-Browder). Since $T-\mu$ is Kato, from [24, Lemma 20.9] it follows that $T-\mu$ is bounded below (surjective). Now, as in the previous part of the proof, we can conclude that $T$ is bounded below (surjective).

Proposition 3.2. Let $T \in L(X)$ and $1 \leq i \leq 12$. If $T$ belongs to the set $\mathbf{g D R}_{i}$, then $0 \notin \operatorname{acc} \sigma_{\mathbf{R}_{i}}(T)$. 
Proof. Let $(M, N) \in \operatorname{Red}(T)$ such that $T_{M} \in \mathbf{R}_{i}$ and $T_{N}$ is quasinilpotent. Since $\mathbf{R}_{i}$ is open, there exists $\epsilon>0$ such that $(T-\lambda)_{M}=T_{M}-\lambda \in \mathbf{R}_{i}$ for $|\lambda|<\epsilon$. On the other hand, $(T-\lambda)_{N}=T_{N}-\lambda \in L(X)^{-1} \subset \mathbf{R}_{i}$ for every $\lambda \neq 0$. Now by applying Lemma 2.2 we obtain that $T-\lambda \in \mathbf{R}_{i}$ for $0<|\lambda|<\epsilon$, and so $0 \notin \operatorname{acc} \sigma_{\mathbf{R}_{i}}(T)$.

We now state the first main result.

Theorem 3.3. Let $T \in L(X)$ and $1 \leq i \leq 6$. The following conditions are equivalent:

(i) There exists $(M, N) \in \operatorname{Red}(T)$ such that $T_{M} \in \mathbf{R}_{i}$ and $T_{N}$ is quasinilpotent, that is $T \in \mathbf{g D R}_{i}$;

(ii) $T$ admits a GKD and $0 \notin \operatorname{acc} \sigma_{\mathbf{R}_{i}}(T)$;

(iii) $T$ admits $a G K D$ and $0 \notin \operatorname{int} \sigma_{\mathbf{R}_{i}}(T)$;

(iv) There exists a projection $P \in L(X)$ that commutes with $T$ such that $T+P \in \mathbf{R}_{i}$ and $T P$ is quasinilpotent.

Proof. (i) $\Longrightarrow$ (ii): Let $T=T_{M} \oplus T_{N}$, where $T_{M} \in \mathbf{R}_{i}$ and $T_{N}$ is quasinilpotent. Then $0 \notin \operatorname{acc} \sigma_{\mathbf{R}_{i}}(T)$ by Proposition 3.2. From [24, Theorem 16.21] it follows that there exist two closed $T$-invariant subspaces $M_{1}$ and $M_{2}$ such that $M=M_{1} \oplus M_{2}, M_{2}$ is finite dimensional, $T_{M_{1}}$ is Kato and $T_{M_{2}}$ is nilpotent. We have $X=M_{1} \oplus\left(M_{2} \oplus N\right), M_{2} \oplus N$ is closed, $T_{M_{2} \oplus N}=T_{M_{2}} \oplus T_{N}$ is quasinilpotent and thus $T$ admits the $\operatorname{GKD}\left(M_{1}, M_{2} \oplus N\right)$.

(ii) $\Longrightarrow$ (iii): Clear.

(iii) $\Longrightarrow$ (i): Let $i \in\{1,2,3\}$. Assume that $T$ admits a GKD and $0 \notin \operatorname{int} \sigma_{\mathbf{R}_{i}}(T)$, that is $0 \in \operatorname{acc} \rho_{\mathbf{R}_{i}}(T)$. Then there exists $(M, N) \in \operatorname{Red}(T)$ such that $T_{M}$ is Kato and $T_{N}$ is quasinilpotent, and also, because of $0 \in$ acc $\rho_{\mathbf{R}_{i}}(T)$, according to Lema 2.2 (i), it follows that $0 \in \operatorname{acc} \rho_{\mathbf{R}_{i}}\left(T_{M}\right)$. From Proposition 3.1(i) it follows that $T_{M} \in \mathbf{R}_{i}$, and so $T \in \mathbf{g D R}_{i}(X)$.

Suppose that $T$ admits a GKD and $0 \notin \operatorname{int} \sigma_{\mathcal{W}_{+}}(T)$, i.e. $0 \in \operatorname{acc} \rho_{\mathcal{W}_{+}}(T)$. Then there exists $(M, N) \in \operatorname{Red}(T)$ such that $T_{M}$ is Kato and $T_{N}$ is quasinilpotent. We show that $0 \in \operatorname{acc} \rho_{\mathcal{W}_{+}}\left(T_{M}\right)$. Let $\epsilon>0$. From $0 \in$ acc $\rho_{\mathcal{W}_{+}}(T)$ it follows that there exists $\lambda \in \mathbb{C}$ such that $0<|\lambda|<\epsilon$ and $T-\lambda \in \mathcal{W}_{+}(X)$. As $T_{N}$ is quasinilpotent, $T_{N}-\lambda$ is invertible, and so, according to Lema 2.2(iii), we conclude that $T_{M}-\lambda \in \mathcal{W}_{+}(M)$, that is $\lambda \in \rho_{\mathcal{W}_{+}}\left(T_{M}\right)$. Therefore, $0 \in \operatorname{acc} \rho_{\mathcal{W}_{+}}\left(T_{M}\right)$ and from Proposition [3.1(ii) it follows that $T_{M}$ is upper semi-Weyl, and so $T \in \mathbf{g D} \mathcal{W}_{+}(X)$. The cases $i=5$ and $i=6$ can be proved similarly.

(i) $\Longrightarrow$ (iv): Suppose that there exists $(M, N) \in \operatorname{Red}(T)$ such that $T_{M} \in \mathbf{R}_{i}$ and $T_{N}$ is quasinilpotent. Let $P \in L(X)$ be a projection such that 
$N(P)=M$ and $R(P)=N$. Then $T P=P T$ and every element $x \in X$ may be represented as $x=x_{1}+x_{2}$, where $x_{1} \in M$ and $x_{2} \in N$. Also,

$$
\left\|(T P)^{n} x\right\|^{\frac{1}{n}}=\left\|T^{n} P x\right\|^{\frac{1}{n}}=\left\|\left(T_{N}\right)^{n} x_{2}\right\|^{\frac{1}{n}} \rightarrow 0(n \rightarrow \infty),
$$

since $T_{N}$ is quasinilpotent. We obtain $H_{0}(T P)=X$, so $T P$ is quasinilpotent. Since $(T+P)_{M}=T_{M}$ and $(T+P)_{N}=T_{N}+I_{N} \in L(N)^{-1}$, where $I_{N}$ is identity on $N$, we have that $(T+P)_{M} \in \mathbf{R}_{i}$ and $(T+P)_{N} \in \mathbf{R}_{i}$ and hence, $T+P \in \mathbf{R}_{i}$ by Lemma 2.2 (i) and (ii).

(iv) $\Longrightarrow$ (i): Assume that there exists a projection $P \in L(X)$ that commutes with $T$ such that $T+P \in \mathbf{R}_{i}$ and $T P$ is quasinilpotent. Put $N(P)=M$ and $R(P)=N$. Then $X=M \oplus N, T(M) \subset M$ and $T(N) \subset N$. For every $x \in N$ we have

$$
\left\|\left(T_{N}\right)^{n} x\right\|^{\frac{1}{n}}=\left\|T^{n} P^{n} x\right\|^{\frac{1}{n}}=\left\|(T P)^{n} x\right\|^{\frac{1}{n}} \rightarrow 0(n \rightarrow \infty),
$$

since $T P$ is quasinilpotent. It follows that $H_{0}\left(T_{N}\right)=N$ and hence, $T_{N}$ is quasinilpotent. It remains to prove that $T_{M} \in \mathbf{R}_{i}$. For $i \in\{1,2,3\}$, by Lemma 2.2(i) we deduce that $T_{M}=(T+P)_{M} \in \mathbf{R}_{i}$. Set $i=4$. Since $T_{N}$ is quasinilpotent, it follows that $T_{N}+I_{N}$ is invertible, where $I_{N}$ is identity on $N$. From $T+P \in \mathcal{W}_{+}(X)$ and the decomposition

$$
T+P=(T+P)_{M} \oplus(T+P)_{N}=T_{M} \oplus\left(T_{N}+I_{N}\right),
$$

according to Lemma 2.2(iii), we conclude that $T_{M} \in \mathcal{W}_{+}(M)$. For $i=5$ and $i=6$ we apply similar consideration.

Q. Jiang and H. Zhong show in [14] that if $T \in L(X)$ admits a GKD, $\sigma_{a p}(T)$ $\left(\sigma_{s u}(T)\right)$ does not cluster at 0 if and only if 0 is not an interior point of $\sigma_{a p}(T)$ $\left(\sigma_{s u}(T)\right)$. In Theorems 3.4 and 3.5 we obtain their result in a different way and also provide further conditions that are equivalent to the previous two.

Theorem 3.4. Let $T \in L(X)$. The following conditions are equivalent:

(i) $H_{0}(T)$ is closed and there exists a closed subspace $M$ of $X$ such that $\left(M, H_{0}(T)\right) \in \operatorname{Red}(T)$ and $T(M)$ is closed;

(ii) There exists $(M, N) \in \operatorname{Red}(T)$ such that $T_{M}$ is bounded below and $T_{N}$ is quasinilpotent, that is $T \in \mathrm{gD} \mathcal{M}(X)$;

(iii) $T$ admits a GKD and $0 \notin \operatorname{acc} \sigma_{a p}(T)$;

(iv) $T$ admits a GKD and $0 \notin \operatorname{int} \sigma_{a p}(T)$;

(v) There exists a bounded projection $P$ on $X$ which commutes with $T$ such that $T+P$ is bounded below and $T P$ is quasinilpotent; 
(vi) There exists $(M, N) \in \operatorname{Red}(T)$ such that $T_{M}$ is upper semi-Browder and $T_{N}$ is quasinilpotent, that is $T \in \mathbf{g D} \mathcal{B}_{+}(X)$;

(vii) $T$ admits a GKD and $0 \notin \operatorname{acc} \sigma_{\mathcal{B}_{+}}(T)$;

(viii) $T$ admits a GKD and $0 \notin \operatorname{int} \sigma_{\mathcal{B}_{+}}(T)$;

(ix) There exists a bounded projection $P$ on $X$ which commutes with $T$ such that $T+P$ is upper semi-Browder and $T P$ is quasinilpotent.

In particular, if $T$ satisfies any of the conditions (i)-(ix), then the subspace $N$ in (ii) is uniquely determined and $N=H_{0}(T)$.

Proof. (i) $\Longrightarrow$ (ii): Suppose that $H_{0}(T)$ is closed and that there exists a closed $T$-invariant subspace $M$ of $X$ such that $X=H_{0}(T) \oplus M$ and $T(M)$ is closed. For $N=H_{0}(T)$ we have that $(M, N) \in \operatorname{Red}(T)$ and $H_{0}\left(T_{N}\right)=N$, which implies that $T_{N}$ is quasinilpotent. From $N\left(T_{M}\right)=N(T) \cap M \subset$ $H_{0}(T) \cap M=\{0\}$ it follows that $T_{M}$ is injective and since $R\left(T_{M}\right)=T(M)$ is a closed subspace in $M$, we conclude that $T_{M}$ is bounded below.

(ii) $\Longrightarrow$ (i): Assume that there exists $(M, N) \in \operatorname{Red}(T)$ such that $T_{M}$ is bounded below and $T_{N}$ is quasinilpotent. Then $(M, N)$ is a GKD for $T$, and so from [1, Corollary 1.69] it follows that $H_{0}(T)=H_{0}\left(T_{M}\right) \oplus H_{0}\left(T_{N}\right)=$ $H_{0}\left(T_{M}\right) \oplus N$. Since $T_{M}$ is bounded below, we get that $H_{0}\left(T_{M}\right)=\{0\}$ and hence $H_{0}(T)=N$. Therefore, $H_{0}(T)$ is closed and complemented with $M$, $\left(M, H_{0}(T)\right) \in \operatorname{Red}(T)$, and $T(M)$ is closed because $T_{M}$ is bounded below.

The implications (ii) $\Longrightarrow$ (iii) and (vi) $\Longrightarrow$ (vii) can be proved analogously to the proof of the implication (i) $\Longrightarrow$ (ii) in Theorem 3.3 . The implications (iii) $\Longrightarrow$ (iv) and (vii) $\Longrightarrow$ (viii) are clear.

(viii) $\Longrightarrow$ (ii): Let $T$ admit a GKD and let $0 \notin \operatorname{int} \sigma_{\mathcal{B}_{+}}(T)$, i.e. $0 \in$ acc $\rho_{\mathcal{B}_{+}}(T)$. There exists $(M, N) \in \operatorname{Red}(T)$ such that $T_{M}$ is Kato and $T_{N}$ is quasinilpotent. From $0 \in \operatorname{acc} \rho_{\mathcal{B}_{+}}(T)$ it follows that $0 \in \operatorname{acc} \rho_{\mathcal{B}_{+}}\left(T_{M}\right)$ according to Lemma $2.2(\mathrm{i})$. From Proposition 3.1(iv) it follows that $T_{M}$ is bounded below, and hence $T \in \mathbf{g D} \mathcal{M}(X)$.

(iv) $\Longrightarrow$ (ii): This implication can be proved by using Proposition 3.1(iii), analogously to the proof of the implication (viii) $\Longrightarrow$ (ii).

(ii) $\Longrightarrow$ (vi): Follows from the fact that every bounded below operator is upper semi-Browder.

The equivalences (v) $\Longleftrightarrow$ (ii) and (vi) $\Longleftrightarrow$ (ix) can be proved analogously to the equivalence (i) $\Longleftrightarrow$ (iv) in Theorem 3.3 .

Theorem 3.5. For $T \in L(X)$ the following conditions are equivalent:

(i) $K(T)$ is closed and there exists a closed subspace $N$ of $X$ such that $N \subset H_{0}(T)$ and $(K(T), N) \in \operatorname{Red}(T)$; 
(ii) There exists $(M, N) \in \operatorname{Red}(T)$ such that $T_{M}$ is surjective and $T_{N}$ is quasinilpotent, that is $T \in \mathbf{g D Q}(X)$;

(iii) $T$ admits a GKD and $0 \notin \operatorname{acc} \sigma_{s u}(T)$;

(iv) $T$ admits $a G K D$ and $0 \notin \operatorname{int} \sigma_{s u}(T)$;

(v) There exists a bounded projection $P$ on $X$ which commutes with $T$ such that $T+P$ is surjective and $T P$ is quasinilpotent;

(vi) There exists $(M, N) \in \operatorname{Red}(T)$ such that $T_{M}$ is lower semi-Browder and $T_{N}$ is quasinilpotent, that is $T \in \mathbf{g D} \mathcal{B}_{-}(X)$;

(vii) $T$ admits $a G K D$ and $0 \notin \operatorname{acc} \sigma_{\mathcal{B}_{-}}(T)$;

(viii) $T$ admits $a G K D$ and $0 \notin \operatorname{int} \sigma_{\mathcal{B}_{-}}(T)$;

(ix) There exists a bounded projection $P$ on $X$ which commutes with $T$ such that $T+P$ is lower semi-Browder and TP is quasinilpotent.

In particular, if $T$ satisfies any of the conditions (i)-(ix), then the subspace $M$ in (ii) is uniquely determined and $M=K(T)$.

Proof. (i) $\Longrightarrow$ (ii): Assume that $K(T)$ is closed and that there exists a closed $T$-invariant subspace $N$, such that $N \subset H_{0}(T)$ and $X=K(T) \oplus N$. For $M=K(T)$ we have that $(M, N) \in \operatorname{Red}(T), R\left(T_{M}\right)=R(T) \cap M=$ $R(T) \cap K(T)=K(T)=M$, and so $T_{M}$ is surjective. Since $H_{0}\left(T_{N}\right)=$ $H_{0}(T) \cap N=N$, we conclude that $T_{N}$ is quasinilpotent.

(ii) $\Longrightarrow$ (i): Suppose that there exists $(M, N) \in \operatorname{Red}(T)$ such that $T_{M}$ is surjective and $T_{N}$ is quasinilpotent. Then $(M, N)$ is a GKD for $T$ and from [1, Theorem 1.41] we obtain that $K(T)=K\left(T_{M}\right)$. Since $T_{M}$ is surjective, it follows that $K\left(T_{M}\right)=M$, and so $K(T)=M$ and $K(T)$ is closed. Thus $(K(T), N) \in \operatorname{Red}(T)$ and since $T_{N}$ is quasinilpotent, we have that $N=$ $H_{0}\left(T_{N}\right) \subset H_{0}(T)$.

The rest of the proof is similar to the proofs of Theorems 3.4 and 3.3 .

The equivalences (i) $\Longleftrightarrow$ (ii) of Theorem 3.4 and Theorem 3.5 were proved recently, see Proposition 3.2 and Proposition 3.4 in [13, but we emphasize that the authors permanently use term "left invertible" instead of "bounded below" and "right invertible" instead of "surjective". Also, the equivalences $(\mathrm{i}) \Longleftrightarrow(\mathrm{v})$ of the theorems mentioned above are established in [10].

Remark 3.6. If $T$ is generalized Drazin invertible, then from Theorem 2.1 and Theorem 3.4 it follows that $T$ is generalized Drazin bounded below and in that case the closed subspace $M$ of $X$ which satisfies the condition (i) in Theorem 3.4, i.e. such that $\left(M, H_{0}(T)\right) \in \operatorname{Red}(T)$ and $T(M)$ is closed, is uniquely determined-we show that it must be equal to $K(T)$. In other words, the projection $P$ which satisfies the condition (v) in Theorem 3.4 is uniquely 
determined-it is equal to the spectral idempotent of $T$ corresponding to the set $\{0\}$.

Indeed, from Theorem 3.4 it follows that $T=T_{M} \oplus T_{H_{0}(T)}, T_{M}$ is bounded below and $T_{H_{0}(T)}$ is quasinilpotent. Since $T$ is generalized Drazin invertible, we have that $0 \notin$ acc $\sigma(T)$, and hence, $0 \notin \operatorname{acc} \sigma\left(T_{M}\right)$. $T_{M}$ is Kato since it is bounded below, and so by Proposition [3.1(i) we obtain that $T_{M}$ is invertible. Since $T$ admits a $\operatorname{GKD}\left(M, H_{0}(T)\right)$, from [1, Theorem 3.15] it follows that $M=K(T)$. The similar observation can be stated in the context of Theorem 3.5 .

In the following theorem we give several necessary and sufficient conditions for $T \in L(X)$ to be generalized Drazin invertible.

Theorem 3.7. Let $T \in L(X)$. The following conditions are equivalent:

(i) $T$ is generalized Drazin invertible;

(ii) $T$ admits a GKD and $0 \notin \operatorname{int} \sigma(T)$;

(iii) $T$ admits a GKD and $0 \notin \operatorname{int} \sigma_{\mathcal{B}}(T)$;

(iv) $T$ admits a $G K D$ and $0 \notin \operatorname{acc} \sigma_{\mathcal{B}}(T)$;

(v) There exists $(M, N) \in \operatorname{Red}(T)$ such that $T_{M}$ is Browder and $T_{N}$ is quasinilpotent;

(vi) There exists a bounded projection $P$ on $X$ which commutes with $T$ such that $T+P$ is Browder and TP is quasinilpotent.

Proof. Similar to the proof of Theorem 3.4

Analysis similar to that in the proof of Theorem 3.3 gives the following result.

Theorem 3.8. Let $T \in L(X)$ and $1 \leq i \leq 12$. The following conditions are equivalent:

(i) There exists $(M, N) \in \operatorname{Red}(T)$ such that $T_{M} \in \mathbf{R}_{i}$ and $T_{N}$ is nilpotent, that is $T \in \mathbf{D R}_{i}$;

(ii) $T$ is of Kato type and $0 \notin \operatorname{acc} \sigma_{\mathbf{R}_{i}}(T)$;

(iii) $T$ is of Kato type and $0 \notin \operatorname{int} \sigma_{\mathbf{R}_{i}}(T)$;

(iv) There exists a projection $P \in L(X)$ that commutes with $T$ such that $T+P \in \mathbf{R}_{i}$ and $T P$ is nilpotent.

Using [5, Theorem 2.7] and [7, Lemma 4.1] we see that if $i=3(i=6)$ then the conditions (i)-(iv) of Theorem 3.8 are equivalent to the fact that $T$ is B-Fredholm ( $T$ is B-Weyl), while if $i=12$ these conditions are equivalent 
to the fact that $T$ is Drazin invertible. Similar to the definitions of the BFredholm and B-Weyl operators, the classes $\mathbf{B R}_{i}$ are introduced and studied [6]. If $i \in\{1,2,4,5,7,8,10,11\}$ then it is possible to show that the conditions (i)-(iv) of Theorem 3.8 are equivalent to the fact that $T$ is of Kato type and $T$ belongs to the class $\mathbf{B R}_{i}$. For the case of a Hilbert space see [6, Theorem $3.12]$.

Corollary 3.9. Let $T \in L(X)$ and let $0 \in \partial \sigma_{\mathbf{R}_{i}}(T), 1 \leq i \leq 12$. Then the following assertions hold.

(i) $T$ admits a generalized Kato decomposition if and only if $T$ belongs to the class $\mathbf{g D R}_{i}$.

(ii) $T$ is of Kato type if and only if $T$ belongs to the class $\mathbf{D R}_{i}$.

Proof. Follows from the equivalence (i) $\Longleftrightarrow$ (iii) in Theorem 3.3 , the equivalences (ii) $\Longleftrightarrow$ (iv) in Theorems 3.4 and 3.5 , the equivalence (i) $\Longleftrightarrow$ (ii) in Theorem 3.7 and from the equivalence (i) $\Longleftrightarrow$ (iii) in Theorem 3.8 .

The case $i=12$ is proved in [2, Theorem 2.9] and [14, Theorem 3.8].

Remark 3.10. We remark that

$$
\begin{gathered}
\Phi_{+}(X) \backslash \mathcal{W}_{+}(X) \subset \mathbf{g D} \Phi_{+}(X) \backslash \mathbf{g D} \mathcal{W}_{+}(X), \\
\Phi_{-}(X) \backslash \mathcal{W}_{-}(X) \subset \mathbf{g D \Phi} \Phi_{-}(X) \backslash \mathbf{g D} \mathcal{W}_{-}(X), \\
\Phi(X) \backslash \mathcal{W}(X) \subset \mathbf{g D \Phi}(X) \backslash \mathbf{g D} \mathcal{W}(X) .
\end{gathered}
$$

Indeed, as the index is locally constant, the set $\Phi_{+}(X) \backslash \mathcal{W}_{+}(X)=\{T \in$ $\Phi(X): \operatorname{ind}(T)>0\}$ is open, which implies that the set $\sigma_{\mathcal{W}_{+}}(T) \backslash \sigma_{\Phi_{+}}(T)=$ $\rho_{\Phi_{+}}(T) \backslash \rho \mathcal{W}_{+}(T)$ is open for every $T \in L(X)$. Suppose that $T \in \Phi_{+}(X) \backslash$ $\mathcal{W}_{+}(X)$. Then $T \in \mathbf{g D} \Phi_{+}(X)$ and $0 \in \sigma_{\mathcal{W}_{+}}(T) \backslash \sigma_{\Phi_{+}}(T)$. There exists $\epsilon>0$ such that $K(0, \epsilon) \subset \sigma_{\mathcal{W}_{+}}(T) \backslash \sigma_{\Phi_{+}}(T)$. Hence, $0 \in \operatorname{acc} \sigma_{\mathcal{W}_{+}}(T)$ and $T \notin \mathbf{g D} \mathcal{W}_{+}(X)$ according to Theorem 3.3. Similarly for the remaining inclusions.

The next example shows that the inclusions $\mathbf{g} \mathbf{D} \mathcal{W}_{+}(X) \subset \mathbf{g D} \Phi_{+}(X), \mathbf{g D} \mathcal{W}_{-}(X)$ $\subset \mathbf{g D} \Phi_{-}(X)$ and $\mathbf{g D} \mathcal{W}(X) \subset \mathbf{g D} \Phi(X)$ can be proper.

Example 3.11. Let $U$ and $V$ be the forward and the backward unilateral shifts defined on $\ell_{p}(\mathbb{N}),(1 \leq p<\infty)$, respectively. The operators $U$ and $V$ are Fredholm, ind $(U)=-1$ and $\operatorname{ind}(V)=1$. Therefore, $U \in \Phi_{-}(X) \backslash$ $\mathcal{W}_{-}(X)$ and $V \in \Phi_{+}(X) \backslash \mathcal{W}_{+}(X)$, and also $U, V \in \Phi(X) \backslash \mathcal{W}(X)$. Hence, according to Remark 3.10, $U \in \mathbf{g D} \Phi_{-}(X) \backslash \mathbf{g D} \mathcal{W}_{-}(X), V \in \mathbf{g D} \Phi_{+}(X) \backslash$ $\operatorname{gD} \mathcal{W}_{+}(X)$ and $U, V \in \mathbf{g D} \mathbf{\Phi}(X) \backslash \mathbf{g D} \mathcal{W}(X)$. 
We also show that the inclusions $\operatorname{gD} \mathcal{M}(X) \subset \operatorname{gD} \mathcal{W}_{+}(X)$ and $\operatorname{gD} \mathcal{Q}(X) \subset$ gD $\mathcal{W}_{-}(X)$ can be proper.

Example 3.12. Let $U$ and $V$ be as in Example 3.11 and let $T=U \oplus V$. Then, according to Lemma $2.2(\mathrm{i}), T$ is Fredholm and $\operatorname{ind}(T)=\operatorname{ind}(U)+$ $\operatorname{ind}(V)=0$. Thus $T$ is Weyl and hence, $T$ is generalized Drazin Weyl. Since $\sigma_{a p}(U)=\sigma_{s u}(V)=\partial \mathbb{D}$ and $\sigma_{s u}(U)=\sigma_{a p}(V)=\mathbb{D}$, where $\mathbb{D}=$ $\{\lambda \in \mathbb{C}:|\lambda| \leq 1\}$, it follows that $\sigma_{a p}(T)=\sigma_{a p}(U) \cup \sigma_{a p}(V)=\mathbb{D}$ and $\sigma_{s u}(T)=\sigma_{s u}(U) \cup \sigma_{s u}(V)=\mathbb{D}$. Therefore, $0 \in \operatorname{acc} \sigma_{a p}(T)$ and $0 \in \operatorname{acc} \sigma_{s u}(T)$ and from Theorems 3.4 and 3.5 it follows that $T$ is neither generalized Drazin bounded below nor generalized Drazin surjective.

\section{Applications}

For $T \in L(X)$ we define the spectra with respect to the sets $\mathbf{g D R}_{i}, 1 \leq i \leq$ 12 , in a classical way,

$$
\sigma_{\mathbf{g D R}_{i}}(T)=\{\lambda \in \mathbb{C}: T-\lambda \notin \mathbf{g D R}\}, \quad 1 \leq i \leq 12 .
$$

From Theorems 3.3, 3.4 and 3.5 it follows that

$$
\begin{aligned}
\sigma_{\mathbf{g D R}_{i}}(T) & =\sigma_{g K}(T) \cup \operatorname{acc} \sigma_{\mathbf{R}_{i}}(T) \\
& =\sigma_{g K}(T) \cup \operatorname{int} \sigma_{\mathbf{R}_{i}}(T), 1 \leq i \leq 12 .
\end{aligned}
$$

From Theorem 3.8 we conclude

$$
\sigma_{B \Phi}(T)=\sigma_{K t}(T) \cup \operatorname{int} \sigma_{\Phi}(T) \text { and } \sigma_{B \mathcal{W}}(T)=\sigma_{K t}(T) \cup \operatorname{int} \sigma_{\mathcal{W}}(T) .
$$

From (4.1) it follows that if $T \in L(X)$ has the property that

$$
\sigma_{\mathbf{R}_{i}}(T)=\partial \sigma_{\mathbf{R}_{i}}(T),
$$

then

$$
\sigma_{g K}(T)=\sigma_{\mathbf{g D R}_{i}}(T), 1 \leq i \leq 12 .
$$

Consequently, if $\sigma(T)$ is at most countable or contained in a line, then $\sigma_{g K}(T)=\sigma_{g D}(T)$. Every self-adjoint, as well as, unitary operator on a Hilbert space have the spectrum contained in a line. The spectrum of polynomially meromorphic operator [16] is at most countable.

Proposition 4.1. Let $T \in L(X)$ and $1 \leq i \leq 12$. The following statements hold:

(i) $\sigma_{\mathbf{g D R}_{i}}(T) \subset \sigma_{\mathbf{R}_{i}}(T) \subset \sigma(T)$;

(ii) $\sigma_{\mathbf{g D R}_{i}}(T)$ is a compact subset of $\mathbb{C}$;

(iii) $\sigma_{\mathbf{R}_{i}}(T) \backslash \sigma_{\mathbf{g D R}_{i}}(T)$ consists of at most countably many isolated points. 
Proof. (i): It is obvious.

(ii): It suffices to prove that $\sigma_{\mathbf{g D R}_{i}}(T)$ is closed since it is bounded by the part (i). If $\lambda_{0} \notin \sigma_{\mathbf{g D R}_{i}}(T)$, then $T-\lambda_{0} \in \mathbf{g D R}_{i}$ and by Proposition 3.2 there exists $\epsilon>0$ such that $T-\lambda_{0}-\lambda \in \mathbf{R}_{i} \subset \mathbf{g D R}_{i}$ for $0<|\lambda|<\epsilon$. It means that open disc of radius $\epsilon$ centered at $\lambda_{0}$ is contained in the complement of $\sigma_{\mathbf{g D R}_{i}}(T)$, so $\sigma_{\mathbf{g D R}_{i}}(T)$ is closed.

(iii): If $\lambda \in \sigma_{\mathbf{R}_{i}}(T) \backslash \sigma_{\mathbf{g D R}_{i}}(T)$, then $\lambda \in \sigma_{\mathbf{R}_{i}}(T)$ and $T-\lambda \in \mathbf{g D R}_{i}$. Applying Proposition 3.2 we obtain that $\lambda \in$ iso $\sigma_{\mathbf{R}_{i}}(T)$, and hence $\sigma_{\mathbf{R}_{i}}(T) \backslash$ $\sigma_{\mathbf{g D R}_{i}}(T)$ consists of at most countably many isolated points.

Corollary 4.2. Let $T \in L(X)$. Then the following inclusions hold:

$$
\begin{array}{rcc}
\operatorname{acc} \sigma_{a p}(T) \backslash \operatorname{acc} \sigma_{\mathcal{B}_{+}}(T) & \subset & \sigma_{g K}(T), \\
\operatorname{acc} \sigma_{s u}(T) \backslash \operatorname{acc} \sigma_{\mathcal{B}_{-}}(T) & \subset & \sigma_{g K}(T), \\
\operatorname{acc} \sigma(T) \backslash \operatorname{acc} \sigma_{\mathcal{B}}(T) & \subset & \sigma_{g K}(T), \\
\operatorname{int} \sigma_{a p}(T) \backslash \operatorname{int} \sigma_{\mathcal{B}_{+}}(T) & \subset & \sigma_{g K}(T), \\
\operatorname{int} \sigma_{s u}(T) \backslash \operatorname{int} \sigma_{\mathcal{B}_{-}}(T) & \subset & \sigma_{g K}(T), \\
\operatorname{int} \sigma(T) \backslash \operatorname{int} \sigma_{\mathcal{B}}(T) & \subset & \sigma_{g K}(T) .
\end{array}
$$

Proof. Follows from the equivalences (iii) $\Longleftrightarrow$ (vii) and (iv) $\Longleftrightarrow$ (viii) in Theorems 3.4 and 3.5 .

Remark 4.3. Let $T \in L(X)$ be a Riesz operator with infinite spectrum. It was shown in [14] that $T$ does not admit a GKD. It is interesting to note that the same follows from Corollary 4.2. Namely, $\sigma_{\mathcal{B}}(T)=\{0\}$ and so $0 \notin \operatorname{acc} \sigma_{\mathcal{B}}(T)$, while $0 \in \operatorname{acc} \sigma(T)$. Therefore, $0 \in \operatorname{acc} \sigma(T) \backslash \operatorname{acc} \sigma_{\mathcal{B}}(T)$ and hence $0 \in \sigma_{g K}(T)$ by Corollary 4.2 .

According to Theorems 3.4 and 3.5 it follows that $T$ is not generalized Drazin bounded below (surjective), while according to Theorem 3.3 it follows that $T$ is not generalized Drazin upper (lower) semi-Fredholm. From $\sigma(T)=\sigma_{a p}(T)=\sigma_{s u}(T)$ and $\sigma_{\mathbf{R}_{i}}(T)=\{0\}, 1 \leq i \leq 9$, it follows that $0 \notin \operatorname{int} \sigma_{\mathbf{R}_{i}}(T)=\emptyset, 1 \leq i \leq 12$. This shows that the condition that the operator admits a GKD in the statements (iv), (vii) and (viii) of Theorems 3.4 and 3.5, as well as in the statements (ii), (iii) and (iv) of Theorem 3.7 and also, in the statements (ii) and (iii) of Theorem 3.3 , can not be omitted.

The following question is natural.

Question 4.4. Does an operator which does not admit a GKD and such that 0 is not an accumulation point of its approximate point (resp. surjective) spectrum exist? If the answer is affirmative, then it means that the condition 
that $T$ admits a GKD in the statements (iii) of Theorems 3.4 and 3.5 can not be omitted.

K. Miloud Hocine et al. [13, Theorems 3.16, 3.17] mentioned that the sufficient condition for $T$ to be a sum of a bounded below (surjective) operator and a quasinilpotent one, is that $0 \in$ iso $\sigma_{a p}(T)\left(0 \in\right.$ iso $\left.\sigma_{s u}(T)\right)$, but they did not give the proof. In [4, Propositions 3.5, 3.7] the authors proved that the condition that $0 \in$ iso $\sigma_{a p}(T)\left(0 \in\right.$ iso $\left.\sigma_{s u}(T)\right)$ implies that $T$ admits a GKD, but their proof is incorrect. Namely, from the assumption $0 \in$ iso $\sigma_{a p}(T)$ they conclude wrongly that $T$ is Kato (by incorrect using [9, Thèoréme 2.1]), and from this fact they obtain that $T$ is bounded below, what is a contradiction-this would mean that for arbitrary bounded linear operator $T$, iso $\sigma_{a p}(T)=\emptyset$, which, of course, is not true.

We give an alternative proof of the inclusion

$$
\partial \sigma(T) \cap \operatorname{acc} \sigma(T) \subset \sigma_{g K}(T)
$$

from Q. Jiang and H. Zhong's paper [14] (see Corollary 3.6 and Theorem 3.8), which does not require any use of the single-valued extension property. What is more, we establish the inclusions of the same type for other spectra.

Theorem 4.5. Let $T \in L(X)$. Then the following inclusions hold:

$$
\partial \sigma_{\mathbf{R}_{i}}(T) \cap \operatorname{acc} \sigma_{\mathbf{R}_{i}}(T) \subset \sigma_{g K}(T), \quad 1 \leq i \leq 12 .
$$

Moreover,

$$
\begin{array}{rc}
\partial \sigma_{\mathcal{B}_{+}}(T) \cap \operatorname{acc} \sigma_{a p}(T) & \subset \sigma_{g K}(T) ; \\
\partial \sigma_{\mathcal{B}_{-}}(T) \cap \operatorname{acc} \sigma_{s u}(T) & \subset \sigma_{g K}(T) ; \\
\partial \sigma_{\mathcal{B}}(T) \cap \operatorname{acc} \sigma(T) & \subset \sigma_{g K}(T) .
\end{array}
$$

Proof. From Theorems 3.7 and 2.1, the equivalence (iii) $\Longleftrightarrow$ (iv) in Theorems 3.4 and 3.5 and the equivalence (ii) $\Longleftrightarrow$ (iii) in Theorem 3.3 it foollows that if $T-\lambda \in L(X)$ admits a GKD, then

$$
\lambda \in \operatorname{int} \sigma_{\mathbf{R}_{i}}(T) \Longleftrightarrow \lambda \in \operatorname{acc} \sigma_{\mathbf{R}_{i}}(T), 1 \leq i \leq 12 .
$$

Therefore, we have the inclusions

$$
\partial \sigma_{\mathbf{R}_{i}}(T) \cap \operatorname{acc} \sigma_{\mathbf{R}_{i}}(T)=\operatorname{acc} \sigma_{\mathbf{R}_{i}}(T) \backslash \operatorname{int} \sigma_{\mathbf{R}_{i}}(T) \subset \sigma_{g K}(T), 1 \leq i \leq 12 .
$$

Suppose that $\lambda \in \partial \sigma_{\mathcal{B}_{+}}(T)$ and $T-\lambda$ admits a GKD. Then $\lambda \notin \operatorname{int} \sigma_{\mathcal{B}_{+}}(T)$ and from the equivalence (viii) $\Longleftrightarrow$ (iii) in Theorem 3.4 we get that $\lambda \notin$ 
acc $\sigma_{a p}(T)$. Therefore, if $\lambda \in \partial \sigma_{\mathcal{B}_{+}}(T) \cap \operatorname{acc} \sigma_{a p}(T)$, then $T-\lambda$ does not admit a GKD, i.e. $\lambda \in \sigma_{g K}(T)$. The remaining inclusions can be proved analogously.

From (4.3) it follows that

$$
\partial \sigma_{\mathbf{R}_{i}}(T) \subset \sigma_{g K}(T) \cup \text { iso } \sigma_{\mathbf{R}_{i}}(T), \quad 1 \leq i \leq 12,
$$

which implies that

$$
\partial \sigma_{\mathbf{R}_{i}}(T) \backslash \sigma_{g K}(T) \subset \text { iso } \sigma_{\mathbf{R}_{i}}(T),
$$

and hence $\partial \sigma_{\mathbf{R}_{i}}(T) \backslash \sigma_{g K}(T)$ consist of at most countably many points.

The connected hull of a compact subset $K$ of the complex plane $\mathbb{C}$, denoted by $\eta K$, is the complement of the unbounded component of $\mathbb{C} \backslash K$ ( $[12$, Definition 7.10.1]). Given a compact subset $K$ of the plane, a hole of $K$ is a bounded component of $\mathbb{C} \backslash K$, and so a hole of $K$ is a component of $\eta K \backslash K$. Generally ([12, Theorem 7.10.3]), for compact subsets $H, K \subset \mathbb{C}$,

$$
\partial H \subset K \subset H \Longrightarrow \partial H \subset \partial K \subset K \subset H \subset \eta K=\eta H .
$$

Evidently, if $K \subseteq \mathbb{C}$ is finite, then $\eta K=K$. Therefore, for compact subsets $H, K \subseteq \mathbb{C}$,

$$
\eta K=\eta H \Longrightarrow(H \text { is finite } \Longleftrightarrow K \text { is finite }),
$$

and in that case $H=K$.

For $\lambda_{0} \in \mathbb{C}$ we set $K\left(\lambda_{0}, \epsilon\right)=\left\{\lambda \in \mathbb{C}:\left|\lambda-\lambda_{0}\right|<\epsilon\right\}$.

Theorem 4.6. Let $T \in L(X)$. Then

$$
\begin{aligned}
& \partial \sigma_{g D \mathcal{M}}(T) \subset \partial \sigma_{g D \mathcal{W}_{+}}(T) \subset \partial \sigma_{g D \Phi_{+}}(T)
\end{aligned}
$$

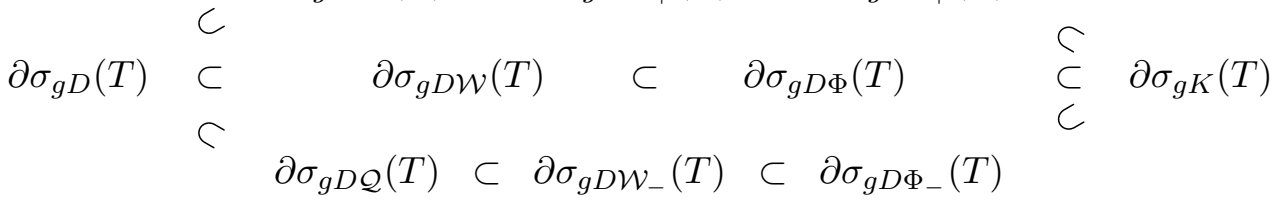

$$
\begin{aligned}
& \partial \sigma_{g D \Phi}(T) \subset \partial \sigma_{g D \Phi_{+}}(T), \quad \partial \sigma_{g D \Phi}(T) \subset \partial \sigma_{g D \Phi_{-}}(T), \\
& \partial \sigma_{g D \mathcal{W}}(T) \subset \partial \sigma_{g D \mathcal{W}_{+}}(T), \quad \partial \sigma_{g D \mathcal{W}}(T) \subset \partial \sigma_{g D \mathcal{W}_{-}}(T),
\end{aligned}
$$

and 


$$
\begin{aligned}
\eta \sigma_{g K}(T) & =\eta \sigma_{g D \Phi_{+}}(T)=\eta \sigma_{g D \mathcal{W}_{+}}(T)=\eta \sigma_{g D \mathcal{M}}(T) \\
& =\eta \sigma_{g D \Phi_{-}}(T)=\eta \sigma_{g D \mathcal{W}_{-}}(T)=\eta \sigma_{g D \mathcal{Q}}(T) \\
& =\eta \sigma_{g D \Phi}(T)=\eta \sigma_{g D \mathcal{W}}(T)=\eta \sigma_{g D}(T)
\end{aligned}
$$

Proof. According to (4.4) it is sufficient to prove the inclusions

(i) $\partial \sigma_{g D}(T) \subset \sigma_{g K}(T) ; \quad$ (ii) $\partial \sigma_{g D \mathcal{M}}(T) \subset \sigma_{g K}(T) ; \quad$ (iii) $\partial \sigma_{g D \mathcal{W}_{+}}(T) \subset$ $\sigma_{g K}(T)$;

(iv) $\partial \sigma_{g D \Phi_{+}}(T) \subset \sigma_{g K}(T) ; \quad(\mathrm{v}) \partial \sigma_{g D \mathcal{Q}}(T) \subset \sigma_{g K}(T) ; \quad\left(\right.$ vi) $\partial \sigma_{g D \mathcal{W}_{-}}(T) \subset$ $\sigma_{g K}(T)$;

(vii) $\partial \sigma_{g D \Phi_{-}}(T) \subset \sigma_{g K}(T) ; \quad($ viii $) \partial \sigma_{g D \mathcal{W}}(T) \subset \sigma_{g K}(T)$; (ix) $\partial \sigma_{g D \Phi}(T) \subset$ $\sigma_{g K}(T)$.

Suppose that $\lambda_{0} \in \partial \sigma_{g D}(T)$. Since $\sigma_{g D}(T)$ is closed, it follows that

$$
\lambda_{0} \in \sigma_{g D}(T)=\sigma_{g K}(T) \cup \operatorname{int} \sigma(T) .
$$

We prove that

$$
\lambda_{0} \notin \operatorname{int} \sigma(T) .
$$

Suppose on the contrary that $\lambda_{0} \in \operatorname{int} \sigma(T)$. Then there exists an $\epsilon>0$ such that $K\left(\lambda_{0}, \epsilon\right) \subset \sigma(T)$. This means that $K\left(\lambda_{0}, \epsilon\right) \subset \operatorname{int} \sigma(T)$ and hence, $K\left(\lambda_{0}, \epsilon\right) \subset \sigma_{g D}(T)$, which contradicts the fact that $\lambda_{0} \in \partial \sigma_{g D}(T)$. Now from (4.7) and (4.8), it follows that $\lambda_{0} \in \sigma_{g K}(T)$.

The inclusions (ii)-(ix) can be proved similarly to the inclusion (i).

From (4.5) and (4.6) it follows that $\sigma_{g K}(T)$ is finite if and only if $\sigma_{\mathbf{g D R}_{i}}(T)$ is finite for any $i \in\{1, \ldots, 12\}$ and in that case $\sigma_{g K}(T)=\sigma_{\mathbf{g D R}_{i}}(T)$ for all $i \in\{1, \ldots, 12\}$. In particular, $\sigma_{g K}(T)$ is finite if and only if $\sigma_{g D}(T)$ is finite, and in that case $\sigma_{g K}(T)=\sigma_{g D}(T)$. Moreover, $\sigma_{g K}(T)=\emptyset$ if and only if $\sigma_{\mathbf{g D R}_{i}}(T)=\emptyset$ for any $i \in\{1, \ldots, 12\}$, that is, if and only if $\sigma(T)$ is a finite set.

The Drazin spectrum of $T \in L(X)$ is defined as

$$
\sigma_{D}(T)=\{\lambda \in \mathbb{C}: T-\lambda \text { is not Drazin invertible }\} .
$$

Theorem 4.7. Let $T \in L(X)$. Then

$$
\partial \sigma_{D}(T) \subset \partial \sigma_{B \mathcal{W}}(T) \subset \partial \sigma_{B \Phi}(T) \subset \partial \sigma_{K t}(T)
$$

and

$$
\eta \sigma_{K t}(T)=\eta \sigma_{B \Phi}(T)=\eta \sigma_{B \mathcal{W}}(T)=\eta \sigma_{D}(T)
$$


Proof. From [2, Theorem 2.9] we have $\sigma_{D}(T)=\sigma_{K t}(T) \cup \operatorname{int} \sigma(T)$. Now use this equality and (4.2), and proceed similarly as in the proof of Theorem 4.6 .

The generalized Drazin resolvent set of $T \in L(X)$ is defined by $\rho_{g K}(T)=$ $\mathbb{C} \backslash \sigma_{g K}(T)$. As a consequence of Theorem 4.6] we get Theorem 3 in [15], which was proved by using the technique based on the single-valued extension property.

Corollary 4.8 ([15], Theorem 3). Let $T \in L(X)$ and let $\rho_{g K}(T)$ has only one component. Then

$$
\sigma_{g K}(T)=\sigma_{g D}(T) .
$$

Proof. Since $\rho_{g K}(T)$ has only one component, it follows that $\sigma_{g K}(T)$ has no holes, and so $\sigma_{g K}(T)=\eta \sigma_{g K}(T)$. From (4.6) it follows that $\sigma_{g D}(T) \supset$ $\sigma_{g K}(T)=\eta \sigma_{g K}(T)=\eta \sigma_{g D}(T) \supset \sigma_{g D}(T)$ and hence $\sigma_{g D}(T)=\sigma_{g K}(T)$.

Theorem 4.9. Let $T \in L(X)$ and $1 \leq i \leq 12$. If

$$
\partial \sigma_{\mathbf{R}_{i}}(T) \subset \operatorname{acc} \sigma_{\mathbf{R}_{i}}(T)
$$

then

$$
\partial \sigma_{\mathbf{R}_{i}}(T) \subset \sigma_{g K}(T) \subset \sigma_{K t}(T) \subset \sigma_{e K}(T) \subset \sigma_{\mathbf{R}_{i}}(T)
$$

and

$$
\eta \sigma_{\mathbf{R}_{i}}(T)=\eta \sigma_{g K}(T)=\eta \sigma_{K t}(T)=\eta \sigma_{e K}(T) .
$$

Proof. From $\partial \sigma_{\mathbf{R}_{i}}(T) \subset \operatorname{acc} \sigma_{\mathbf{R}_{i}}(T)$ it follows that $\partial \sigma_{\mathbf{R}_{i}}(T) \cap \operatorname{acc} \sigma_{\mathbf{R}_{i}}(T)=$ $\partial \sigma_{\mathbf{R}_{i}}(T)$, and so from (4.3) it follows that $\partial \sigma_{\mathbf{R}_{i}}(T) \subset \sigma_{g K}(T)$. (4.11) follows from (4.10) and (4.4).

The Goldberg spectrum of $T \in L(X)$ is defined by

$$
\sigma_{e c}(T)=\{\lambda \in \mathbb{C}: R(T-\lambda) \text { is not closed }\} .
$$

Obviously, $\sigma_{e c}(T) \subset \sigma_{\mathbf{R}_{i}}(T)$ for $1 \leq i \leq 12$.

Theorem 4.10. Let $T \in L(X)$ and $1 \leq i \leq 12$. If

$$
\sigma_{\mathbf{R}_{i}}(T)=\partial \sigma_{\mathbf{R}_{i}}(T)=\operatorname{acc} \sigma_{\mathbf{R}_{i}}(T),
$$

then

$$
\sigma_{e c}(T) \subset \sigma_{g K}(T)=\sigma_{K t}(T)=\sigma_{e K}(T)=\sigma_{\mathbf{R}_{i}}(T)=\sigma_{\mathbf{g D R}_{i}}(T) .
$$


Proof. Suppose that $\sigma_{\mathbf{R}_{i}}(T)=\partial \sigma_{\mathbf{R}_{i}}(T)$ and that every $\lambda \in \sigma_{\mathbf{R}_{i}}(T)$ is not isolated in $\sigma_{\mathbf{R}_{i}}(T)$. From Theorem 4.9 it follows that

$$
\sigma_{\mathbf{R}_{i}}(T)=\partial \sigma_{\mathbf{R}_{i}}(T) \subset \sigma_{g K}(T) \subset \sigma_{K t}(T) \subset \sigma_{e K}(T) \subset \sigma_{\mathbf{R}_{i}}(T),
$$

and so

$$
\sigma_{e c}(T) \subset \sigma_{\mathbf{R}_{i}}(T)=\sigma_{g K}(T)=\sigma_{K t}(T)=\sigma_{e K}(T)=\sigma_{\mathbf{g D R}_{i}}(T) .
$$

Example 4.11. Let $U$ and $V$ be as in Example 3.11. Since $\sigma_{a p}(U)=$ $\sigma_{s u}(V)=\partial \mathbb{D}$, then $\sigma_{a p}(U)=\partial \sigma_{a p}(U)=\operatorname{acc} \sigma_{a p}(U)$ and $\sigma_{s u}(V)=\partial \sigma_{s u}(V)=$ acc $\sigma_{s u}(V)$. From Theorem 4.10 we get

$$
\begin{gathered}
\sigma_{g K}(U)=\sigma_{K}(U)=\sigma_{a p}(U)=\sigma_{g D \mathcal{M}}(U)=\sigma_{g D \mathcal{W}_{+}}(U)=\sigma_{g D \Phi_{+}}(U)=\partial \mathbb{D} \\
\sigma_{g K}(V)=\sigma_{K}(V)=\sigma_{s u}(V)=\sigma_{g D \mathcal{Q}}(V)=\sigma_{g D \mathcal{W}_{-}}(V)=\sigma_{g D \Phi_{-}}(V)=\partial \mathbb{D} .
\end{gathered}
$$

It is not difficult to see that if $K \subset \mathbb{C}$ is compact, then for $\lambda \in \partial K$ there is equivalence: $\lambda \in$ iso $K \Longleftrightarrow \lambda \in$ iso $\partial K$, that is,

$$
\lambda \in \operatorname{acc} K \Longleftrightarrow \lambda \in \operatorname{acc} \partial K \text {. }
$$

Moreover, it is well known that

$$
\begin{aligned}
& \partial \sigma(T) \subset \partial \sigma_{a p}(T) \subset \sigma_{a p}(T), \\
& \partial \sigma(T) \subset \partial \sigma_{s u}(T) \subset \sigma_{s u}(T) .
\end{aligned}
$$

Now, as consequences we get Theorem 3.12 and Corollary 3.13 in [14, as well as Theorems 3.8 and 3.9 in [3].

Corollary $4.12\left([14,[3])\right.$. Let $T \in L(X)$ be an operator for which $\sigma_{a p}(T)=$ $\partial \sigma(T)$ and every $\lambda \in \partial \sigma(T)$ is not isolated in $\sigma(T)$. Then

$$
\sigma_{e c}(T) \subset \sigma_{a p}(T)=\sigma_{g K}(T)=\sigma_{K t}(T)=\sigma_{e K}(T)=\sigma_{K}(T) .
$$

Proof. From $\sigma_{a p}(T)=\partial \sigma(T)$ and (4.15) it follows that $\sigma_{a p}(T)=\partial \sigma_{a p}(T)$, while from (4.14) it follows that every $\lambda \in \partial \sigma(T)$ is not isolated in $\partial \sigma(T)$, i.e. in $\sigma_{a p}(T)$. Now from Theorem 4.10 we get (4.17).

Corollary $4.13\left([14,,[3])\right.$. Let $T \in L(X)$ be an operator for which $\sigma_{s u}(T)=$ $\partial \sigma(T)$ and every $\lambda \in \partial \sigma(T)$ is not isolated in $\sigma(T)$. Then $\sigma_{e c}(T) \subset \sigma_{s u}(T)=$ $\sigma_{g K}(T)=\sigma_{K t}(T)=\sigma_{e K}(T)=\sigma_{K}(T)$.

Proof. Follows from (4.16), (4.14) and Theorem 4.10, analogously to the proof of Corollary 4.12 . 


\section{References}

[1] P. Aiena, Fredholm and local spectral theory, with applications to multipliers, Kluwer Academic Publishers (2004).

[2] P. Aiena, E. Rosas, Single-valued extension property at the points of the approximate point spectrum, J. Math. Anal. Appl. 279 (2003), 180-188.

[3] M. Benharrat and B. Messirdi, On the generalized Kato spectrum, Serdica Math. J. 37 (2011), 283-294.

[4] M. Benharrat, K. Hocine, B. Messirdi, Left and right generalized Drazin invertible operators and local spectral theory, http://arxiv.org/abs/1512.02623

[5] M. Berkani, On a class of quasi-Fredholm operators, Integr. Equ. Oper. Theory 34 (1999), 244-249.

[6] M. Berkani, Restiction of an operator to the range of its powers, Studia Math. 140 (2000), no. 2, 163-175.

[7] M. Berkani, Index of B-Fredholm operators and generalization of a Weyl theorem, Proc. Amer. Math. Soc. 130 (2002), 1717-1723.

[8] E. Boasso, Isolated spectral points and Koliha-Drazin invertible elements in quotient Banach algebras and homomorphism ranges, Math. Proc. Royal Irish Acad. 115A (2015), no.2, 1-15.

[9] W. Bouamama, Les opérateurs pseudo-Fredholm dans les espaces de Banach, Rend. Circ. Math. Palermo, Serie II, Tomo LIII (2004), 313324 .

[10] M. D. Cvetković, On upper and lower generalized Drazin invertible operators, Funct. Anal. Approx. Comp. 7 (2015), 67-74.

[11] A. Dajić and J. J. Koliha, The $\sigma g$-Drazin inverse and the generalized Mbekhta Decomposition, Integr. Equ. Oper. Theory 57 (2007), 309-326.

[12] R.E. Harte, Invertibility and Singularity for Bounded Linear Operators, Marcel Dekker, Inc., New York/Basel, 1988.

[13] K. Hocine, M. Benharrat, B. Messirdi, Left and right generalized Drazin invertible operators, Linear and Multilinear Algebra, 63 (2015) 16351648 . 
[14] Q. Jiang, H. Zhong, Generalized Kato decomposition, single-valued extension property and approximate point spectrum, J. Math. Anal. Appl. 356 (2009), 322-327.

[15] Q. Jiang, H. Zhong, Components of generalized Kato resolvent set and single-valued extension property, Front. Math. China 7 (2012), no. 4, 695-702.

[16] M. A. Kaashoek, M. R. F. Smyth, On operators $T$ such that $f(T)$ is Riesz or meromorphic, Proc. Roy. Irish Acad. Sect. A 72 (1972), 81-87.

[17] M. A. Kaashoek, Ascent, descent, nullity and defect, a note on a paper by A. E. Taylor, Math. Annalen, 172 (1967), 105-115.

[18] C. King, A note on Drazin inverses, Pacific J. Math. 70 (1977), 383390.

[19] J. J. Koliha, A generalized Drazin inverse, Glasgow Math. J. 38 (1996), $367-381$.

[20] J. J. Koliha, Isolated spectral points, Proc. Amer. Math. Soc. 124 (1996), no. 11, 3417-3424.

[21] H. Kroh and P. Volkman, Störungssätze für Semifredholmoperatoren, Math. Zeit. 148 (1976), 295-297.

[22] D. C. Lay, Spectral analysis using ascent, descent, nullity and defect, Math. Ann. 184 (1970), 197-214.

[23] M. Mbekhta, Généralisation de la décomposition de Kato aux opérateurs paranormaux et spectraux, Glasgow Math. J. 29 (1987), 159175.

[24] V. Müller, Spectral theory of linear operators and spectral systems in Banach algebras, Birkhäuser (2007).

[25] C. Schmoeger, On isolated points of the spectrum of a bounded linear operator, Proc. Amer. Math. Soc. 117 (1993), 715-719.

[26] H. Zariouh, H. Zguitti, On pseudo B-Weyl operators and generalized Drazin invertibility for operator matrices, Linear and Multilinear algebra, DOI: 10.1080/03081087.2015.1082959 
Miloš D. Cvetković

University of Niš

Faculty of Sciences and Mathematics

P.O. Box 224, 18000 Niš, Serbia

E-mail: miloscvetkovic83@gmail.com

Snežana Č. Živković-Zlatanović

University of Niš

Faculty of Sciences and Mathematics

P.O. Box 224, 18000 Niš, Serbia

E-mail: mladvlad@mts.rs 